\title{
Optimization of synthetic conditions CMC-g-poly (acrylic acid)/Celite composite superabsorbent by Taguchi method and determination of its absorbency under load
}

\author{
A. Pourjavadi , M. S Amini-Fazl, M. Ayyari \\ Polymer Research Laboratory, Department of Chemistry, Sharif University of Technology, Azadi Ave., \\ P.O.Box 11365-9516, Tehran, Iran
}

Received 21 April 2007; accepted in revised form 17 June 2007

\begin{abstract}
A novel biopolymer-based composite hydrogel was synthesized through chemical crosslinking by graft copolymerization of partially neutralized acrylic acid onto the carboxymethyl cellulose (CMC). The Taguchi method, a robust experimental design, was employed for the optimization of the synthesis based on the swelling capacity of the hydrogels. This method was applied for the experiments and standard L16 orthogonal array with five factors and four levels. In the synthesis of the composite superabsorbent, N,N'-methylene bisacrylamide (MBA) as crosslinker, ammonium persulfate (APS) as initiator, acrylic acid (AA) as monomer, CMC/Celite weight ratio and neutralization percent (NU) were used as important factors. From the analysis of variance of the test results, the most effective factor controlling equilibrium swelling capacity was obtained and maximum water absorbency of the optimized final product was found to be $310 \mathrm{~g} / \mathrm{g}$. The surface morphology of the gel was examined using scanning electron microscopy. Furthermore in this research, swelling capacity of composite SAPs was determined under realistic condition (saline solution absorbency under load).
\end{abstract}

Keywords: polymer gels, Taguchi method, absorbency under load

\section{Introduction}

Superabsorbent polymer (SAP) hydrogels are special polymeric materials that can absorb large amounts of water, saline solutions or physiological fluids as high as 10-1000 times their own weight due to a considerable amount of hydrophilic groups in their structure. The super-swelling characteristics of SAPs make them ideal for use in water absorbing applications such as disposable diapers, feminine napkins, and agriculture, cosmetic and absorbent pads [1-3]. Because of their exceptional properties, i. e. biocompatibility, biodegradability, renewability, and non-toxicity, polysaccharides and proteins are the main part of the natural-based superabsorbent hydrogels. The higher production cost and low gel strength of these superabsorbents, however, restrict their wide application. To improve these limitations, inorganic compounds with low cost can be used. The introduction of inorganic fillers to a polymer matrix increases its strength and stiffness properties. Recently, much attention has been paid to inorganic materials for the preparation of superabsorbent composites, such as montmorillonite $[4,5]$, attapulgite $[6,7]$, kaolin $[8,9]$, mica $[10,11]$, bentonite and sercite $[12,13]$. Mineral powders of Celite are hydrated layered aluminosilicates with reactive $-\mathrm{OH}$ groups on the surface. Celite powder can be dispersed to some extent in water and cross-linked with acrylic acid and $\mathrm{CMC}$. The interaction of mineral powders, reactive site of natural polymers and monomers result in a superabsorbent composite.

*Corresponding author, e-mail: purjavad@ sharif.edu

(C) BME-PT and GTE 
To optimize the affecting variables on swelling capacity of SAPs, Taguchi method can be used [14]. The Taguchi method [15] is a powerful design of experiments tool developed by G. Taguchi. It provides a simple, efficient, and systematic approach to optimize designs for performance, quality, and cost. The parameter design is the key step in the Taguchi method to achieve high quality without increase in cost and the same is adopted in this article. The evaluation of results has been standardized by this method, which can easily be applied by researchers. Among other advantages of the Taguchi method, one can name the possibility of performing experiments in a parallel form [16-19].

There are few studies on the swollen gel strength consideration. When the superabsorbents are under load, the swelling capacity is decreased, so another parameter, i. e. absorbency under load (AUL) is often defined and reported especially in technical data. Although the values of load-free absorbency (free swelling) are usually given in the basic scientific literature, the more realistic values, i. e. AUL are often reported in the technical data sheets and patent articles [20]. Since AUL values are logically changed in proportion to mechanical strength of the swollen gel, AUL can be considered as a measure of the gel strength of SAPs. So, many efforts have been made to achieve superabsorbents having higher AUL or higher strength of the swollen gel [20-22].

Achieving the composite hydrogels with high absorption capabilities has been attempted by researchers. Therefore, optimization of the operational conditions for synthesis in order to prepare samples with high water absorbency is of high importance. Therefore, in this work, optimization of synthesis conditions of a superabsorbent composites based on CMC in the presence of Celite particles to achieve maximum water absorbency was performed by Taguchi method. Also swelling capacity of composite SAPs was determined under realistic conditions (saline solution absorbency under load).

\section{Experimental}

\subsection{Materials}

The polysaccharide carboxymethyl cellulose (CMC) with a degree of substitution (D.S) 0.52 was purchased from Merck and Celite (from Khorassan
Co., Iran,) N,N'-methylene bisacrylamide (MBA, from Fluka), ammonium persulfate (APS, from Fluka), acrylic acid (AA, from Merck) were of analytical grade and used without further purification. All other chemicals were also analytical grade. Double distilled water was used for the hydrogel preparation and swelling measurements.

\subsection{Instrumental analysis}

Samples were characterized as $\mathrm{KBr}$ pellets using a Mattson-1000 FTIR spectrophotometer. Morphology of the dried gel structures was studied by scanning electron microscopy (SEM). Dried superabsorbent powders were coated with thin layer gold and imaged in a SEM instrument (Leo, 1455 VP).

\subsection{Experimental design}

\subsubsection{Selection of factors and their levels}

According to the basic knowledge of composite hydrogels, the concentration of crosslinking agent (MBA), initiator (APS) and monomer (AA), and neutralization percent (NU), CMC/Celite weight ratio are the key synthesis factors affecting the final swelling properties of the hydrogels. These parameters were varied at four levels as shown in Table 1. The applied ranges of the variables were chosen based on the literature and our preliminary experiments.

Table 1. Experimental control factors and their levels

\begin{tabular}{|l|c|c|c|c|}
\hline Control factors & Level-1 & Level-2 & Level-3 & Level-4 \\
\hline MBA [mol/l] & 0.004 & 0.009 & 0.016 & 0.048 \\
\hline APS [mol/1] & 0.005 & 0.010 & 0.016 & 0.021 \\
\hline CMC/Celite & $1 / 0.25$ & $0.75 / 0.5$ & $0.5 / 0.75$ & $0.25 / 1$ \\
\hline AA [mol/l] & 0.60 & 0.92 & 1.23 & 1.54 \\
\hline NU \% & 20 & 50 & 70 & 100 \\
\hline
\end{tabular}

\subsubsection{Selection of orthogonal array and assignment of factors}

Standard tables known as orthogonal arrays (OA) are used to design the experiments in the Taguchi method. An OA with a four level and five factors are shown in Table 2. This OA is particularly designed with the symbol of L16. Each row in the array represents a trial condition with the factor levels, which are indicated by the numbers in the row. The columns correspond to the factors specified in this study, and each column contains four level 1, four level 2 , four level 3 , and four level 4 condi- 
Table 2. Experimental layout of an L16 orthogonal array according to Taguchi's suggestion

\begin{tabular}{|c|c|c|c|c|c|}
\hline \multirow{2}{*}{ Trial number } & \multicolumn{5}{|c|}{ Factors and their levels } \\
\cline { 2 - 6 } & F1 & F2 & F3 & F4 & F5 \\
\hline 1 & 1 & 1 & 1 & 1 & 1 \\
\hline 2 & 1 & 2 & 2 & 2 & 2 \\
\hline 3 & 1 & 3 & 3 & 3 & 3 \\
\hline 4 & 1 & 4 & 4 & 4 & 4 \\
\hline 5 & 2 & 1 & 2 & 3 & 4 \\
\hline 6 & 2 & 2 & 1 & 4 & 3 \\
\hline 7 & 2 & 3 & 4 & 1 & 2 \\
\hline 8 & 2 & 4 & 3 & 2 & 1 \\
\hline 9 & 3 & 1 & 3 & 4 & 2 \\
\hline 10 & 3 & 2 & 4 & 3 & 1 \\
\hline 11 & 3 & 3 & 1 & 2 & 4 \\
\hline 12 & 3 & 4 & 2 & 1 & 3 \\
\hline 13 & 4 & 1 & 4 & 2 & 3 \\
\hline 14 & 4 & 2 & 3 & 1 & 4 \\
\hline 15 & 4 & 3 & 2 & 4 & 1 \\
\hline 16 & 4 & 4 & 1 & 3 & 2 \\
\hline
\end{tabular}

tions (a total of 16 conditions) for the factors assigned to the column. Therefore, the evaluation of results has been standardized by this method, which can easily be applied by researchers. In this article, the results were analyzed statistically by the analysis of variance (ANOVA) method using Qualitek-4 software.

\subsection{Graft copolymerization}

Various amounts of CMC $(0.25-1 \mathrm{~g})$ were dissolved in $25 \mathrm{ml}$ distilled water and were added to a three-necked reactor equipped with a mechanical stirrer (Heidolph RZR 2021, three blade propeller type, $200 \mathrm{rpm}$ ). The reactor was immersed in a thermostatted water bath preset at $80^{\circ} \mathrm{C}$. After complete dissolution of CMC, various amounts of Celite powder $(0.25-1 \mathrm{~g}$ in $5 \mathrm{ml}$ water $)$ were added to the solution and allowed to stir for $10 \mathrm{~min}$. Then, certain amounts of AA $(2-5 \mathrm{ml})$ and MBA (0.03-0.4 $\mathrm{g}$ in $5 \mathrm{ml}$ water) were added to the reaction mixture and allowed to stir for $5 \mathrm{~min}$. Then the initiator solution (0.05-0.2 g APS in $5 \mathrm{ml}$ water) was added to the mixture. After stirring for $5 \mathrm{~min}$, the completion of the reaction, to neutralize (20-100\%) acrylic groups, appropriate amount of $\mathrm{NaOH}(0.22-2.75 \mathrm{gr}$ in $5 \mathrm{ml}$ water) was added. The obtained gel was poured to excess nonsolvent ethanol $(200 \mathrm{ml})$ and remained for $3 \mathrm{~h}$ to dewater. Then ethanol was decanted, and the product was cut into small pieces (diameter $5 \mathrm{~mm}$ ). Again, $200 \mathrm{ml}$ fresh ethanol was added, and the hydrogel was stored for a week. The dried gel particles were filtered and placed in an oven at $55^{\circ} \mathrm{C}$ for a week. After grinding, the powdered composite superabsorbent hydrogel was stored away from moisture, heat, and light.

\subsection{Swelling measurements}

An accurately weighed sample $(0.1 \pm 0.01 \mathrm{~g})$ of the powdered composite with average particle sizes between 40 and 60 mesh $(250-400 \mu \mathrm{m})$ was immersed in $200 \mathrm{ml}$ distilled water for $3 \mathrm{~h}$. The equilibrium swelling (ES) capacity was measured at room temperature by 'tea bag' method and using the Equation (1):

ES $[\mathrm{g} / \mathrm{g}]=\frac{\text { weight of swollen gel }- \text { weight of dried gel }}{\text { weight of dried gel }}$

\subsection{Measurement of AUL}

A macro-porous sintered glass filter plate (porosity \# $0, d=80 \mathrm{~mm}, h=7 \mathrm{~mm}$ ) was placed in a Petri dish $(d=118 \mathrm{~mm}, h=12 \mathrm{~mm})$, and a weighed, dried SAP sample $(0.1 \pm 0.01 \mathrm{~g})$ was uniformly placed on the surface of a polyester gauze located on the sintered glass. A cylindrical solid weight (Teflon, $d=60 \mathrm{~mm}$, variable height) which could slip freely in a glass cylinder $(d=60 \mathrm{~mm}, h=$ $50 \mathrm{~mm}$ ) was used to apply the desired load (applied pressure $6205 \mathrm{~Pa}$ ) to the dry SAP particles Figure 1 . Then, $0.9 \%$ saline solution was added so that the liquid level was equal to the height of the sintered glass filter. The dish and its contents were covered to prevent surface evaporation and probable change in the saline concentration. After

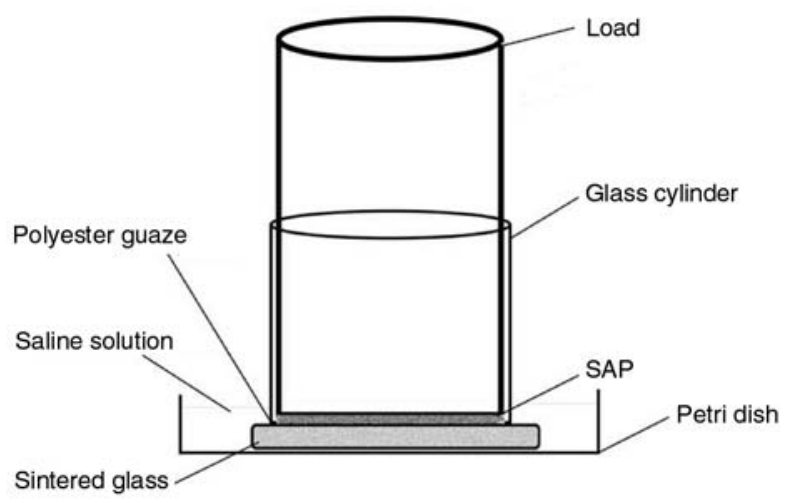

Figure 1. Scheme of the absorbency under load (AUL) tester 
$150 \mathrm{~min}$, the swollen particles were weighed again and AUL was calculated using the Equation (2):

AUL $[\mathrm{g} / \mathrm{g}]=\frac{W_{2}-W_{1}}{W_{1}}$

where $W_{1}$ and $W_{2}$ denote the weight of dry superabsorbent and swollen hydrogel, respectively.

\section{Results and discussion}

\subsection{Synthesis and characterization}

Graft copolymerization of acrylic acid onto carboxymethyl cellulose was carried out in the presence of a crosslinking agent (MBA), powdery Celite and ammonium persulfate as an initiator. The persulfate decomposes on heating and produces sulfate anion-radicals that abstract hydrogen atoms from the hydroxyl groups of CMC backbones. This redox system results in active centers capable of radically initiating the polymerization of acrylic acid, leading to a graft copolymer. Since a crosslinking agent (MBA) is present in the system, the copolymer comprises a crosslinked structure. Celite powder cross-links with the acrylic acid and the CMC. The carboxylate groups of the grafted poly (acrylic acid) can react with the $-\mathrm{OH}$ groups on the Celite surface. The substitution of $-\mathrm{OH}$ groups in the surface of Celite by carboxylate groups results in the ester formation. The clay in the polymerization reaction can be considered as acting in one or both of two ways: a) The Celite particles act as a crosslinking agent. This means that the carboxylate groups of the sodium poly (acrylate) chains react with Celite. b) Celite particles prevent the polymer chains from growing by a chain transfer mechanism [10].

\subsubsection{Infrared spectroscopic analysis}

FT-IR spectroscopy was used for identify the product. The FT-IR spectra of the initial substrates and CMC-g-poly (sodium acrylate)/Celite composite are depicted in Figure 2. Figure 2a represents the spectrum of Celite. The bands at $1080 \mathrm{~cm}^{-1}$ could be attributed to the stretching of the siloxane (-Si-O-Si-) group, and this was confirmed by the $-\mathrm{Si}-\mathrm{O}-\mathrm{Si}-$ bending vibration at $481 \mathrm{~cm}^{-1}$. The band at $790 \mathrm{~cm}^{-1}$ was attributed to $\mathrm{SiO}-\mathrm{H}$ vibration $[23,24]$.
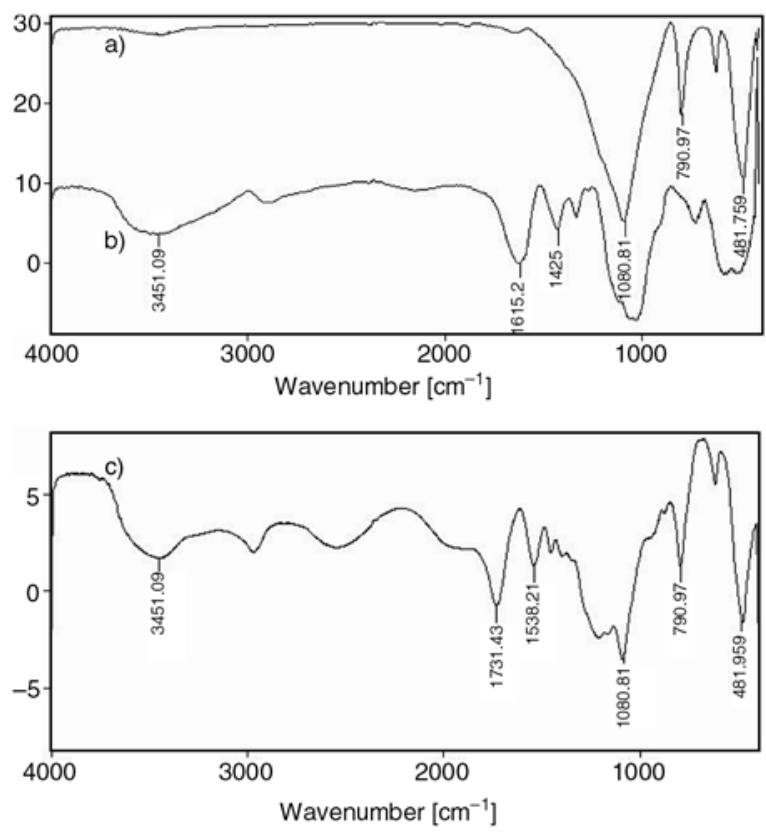

Figure 2. FT-IR spectra of (a) Celite, (b) CMC, (c) CMC$g$-poly (sodium acrylate)/Celite composite

In the spectrum of CMC Figure $2 \mathrm{~b}$ two strong peaks were observed at 1615 and $1425 \mathrm{~cm}^{-1}$ due to the asymmetrical and symmetrical stretching of $\mathrm{COO}^{-}$groups. Characteristic absorption peak of CMC appeared at $3451 \mathrm{~cm}^{-1}$ for the hydroxyl group.

Figure 2c shows the FT-IR spectrum of CMC- $g$ poly (sodium acrylate)/Celite composite. Two new absorption peaks appeared at 1731 and $1538 \mathrm{~cm}^{-1}$ may be attributed to the reaction between carboxylate groups of CMC and acrylic acid with hydroxyl groups on Celite.

\subsection{Optimization of the grafting conditions}

The relationship between the swelling ratio and network structure parameters given by Flory [25] is usually used as the Equation (3):

$q_{m}^{5 / 3}=\frac{\left(\frac{i}{2 v_{u} S^{* 1 / 2}}\right)^{2}+\frac{\frac{1}{2}-\chi_{1}}{v_{1}}}{\frac{v_{e}}{V_{0}}}$

where $q_{m}$ is swelling ratio; $i / v_{u}$ is the concentration of the fixed charge of the unswollen networks; $S^{*}$ is the ionic strength of the swollen solution; $v_{e} / V_{0}$ is the crosslinking density which refers to the number of effectively crosslinked chains in unit volume. 
The term $\left(1 / 2-\chi_{1}\right) / v_{1}$ stands for the networkmedium affinity.

According to Equation (3), there are many variables affecting the ultimate swelling capacity. Some of these variables have been selected in this research. These parameters and the related levels are presented in Table 1. After selecting the mentioned variables, an OA table was formed by Qualitek-4 software (Table 2). The experimental layout after assigning the values of the parameters is shown in Table 3. According to Table 3, 16 experiments were carried out and the ES capacities were measured. The test results are shown in Table 4. Having used the Qualitek-4 software, the optimized circumstances and the contribution of each factor are obtained by ANOVA analysis. It should be emphasized that the interaction between the variables were neglected. The optimized values of the ES capacity, MBA, APS, AA concentration, $\mathrm{CMC} /$ Celite and NU \% are $310 \mathrm{~g} / \mathrm{g}, 0.004 \mathrm{~mol} / \mathrm{l}$, $0.016 \mathrm{~mol} / \mathrm{l}, 1.54 \mathrm{~mol} / \mathrm{l}, 0.25 / 1 \mathrm{gr}, 50 \%$ respectively.

After conducting the synthesis of hydrogel with above optimized reaction composition for three times and measuring the ES capacities, following data were obtained: 315, 310, 308 (g water/g dried gel). Observed slight errors can be attributed to applied materials and apparatus for the synthesis.

Table 3. Experimental layout after assigning the values of the parameters

\begin{tabular}{|c|c|c|c|c|c|}
\hline Trial & MBA & APS & CMC/Celite & AAc & Nu \\
\hline 1 & 0.004 & 0.005 & $1 / 0.25$ & 0.60 & 20 \\
\hline 2 & 0.004 & 0.010 & $0.75 / 0.5$ & 0.92 & 50 \\
\hline 3 & 0.004 & 0.016 & $0.5 / 0.75$ & 1.23 & 70 \\
\hline 4 & 0.004 & 0.021 & $0.25 / 1$ & 1.54 & 100 \\
\hline 5 & 0.009 & 0.005 & $0.75 / 0.5$ & 1.23 & 100 \\
\hline 6 & 0.009 & 0.010 & $1 / 0.25$ & 1.54 & 70 \\
\hline 7 & 0.009 & 0.016 & $0.25 / 1$ & 0.60 & 50 \\
\hline 8 & 0.009 & 0.021 & $0.5 / 0.75$ & 0.92 & 20 \\
\hline 9 & 0.016 & 0.005 & $0.5 / 0.75$ & 1.54 & 50 \\
\hline 10 & 0.016 & 0.010 & $0.25 / 1$ & 1.23 & 20 \\
\hline 11 & 0.016 & 0.016 & $1 / 0.25$ & 0.92 & 100 \\
\hline 12 & 0.016 & 0.021 & $0.75 / 0.5$ & 0.60 & 70 \\
\hline 13 & 0.048 & 0.005 & $0.25 / 1$ & 0.92 & 70 \\
\hline 14 & 0.048 & 0.010 & $0.5 / 0.75$ & 0.60 & 100 \\
\hline 15 & 0.048 & 0.016 & $0.75 / 0.5$ & 1.54 & 20 \\
\hline 16 & 0.048 & 0.021 & $1 / 0.25$ & 1.23 & 50 \\
\hline
\end{tabular}

The contribution of factors according to ANOVA table derived for this study is as shown in Table 5. It can be seen from the ANOVA results that the most effective factor is MBA concentration.

To determine the swelling capacity of composite SAPs under realistic condition, gels of differents water absorbance capacity were used, selected samples exhibited heighten absorbency (optimized final product), medium absorbency (sample 6), and the lowest absorbency (sample 16) that had already absorbed saline solution under $6205 \mathrm{~Pa}$ load.

As shown in Figure 3. The minimum time needed for the highest AUL in the case of each load was determined to be $150 \mathrm{~min}$. After this time, the AUL values remained unchanged. In addition, AUL decreases with the increase in loading, as expected (data are not shown). The most important factor among these forces is the ionic interaction due to the presence of mobile ions such as $\mathrm{Na}^{+}$and $\mathrm{K}^{+}$in the superabsorbent network structure. These mobile ions cannot leave the network due to the charge equilibrium in the gel phase. The presence

Table 5. The results of analysis of variance

\begin{tabular}{|l|c|c|c|}
\hline \multicolumn{1}{|c|}{ Factor } & $\begin{array}{c}\text { Degree of } \\
\text { freedom }\end{array}$ & $\begin{array}{c}\text { Sum. } \\
\text { of square }\end{array}$ & $\begin{array}{c}\text { Contribution of } \\
\text { factor [\%] }\end{array}$ \\
\hline MBA [mol/l] & 1 & 51881.25 & 57.09 \\
\hline APS [mol/l] & 3 & 7108.25 & 7.93 \\
\hline CMC/Celite [g/g] & 4 & 14394.75 & 16.06 \\
\hline AA [mol/l] & 4 & 4551.25 & 5.07 \\
\hline NU \% & 2 & 11666.25 & 13.02 \\
\hline
\end{tabular}

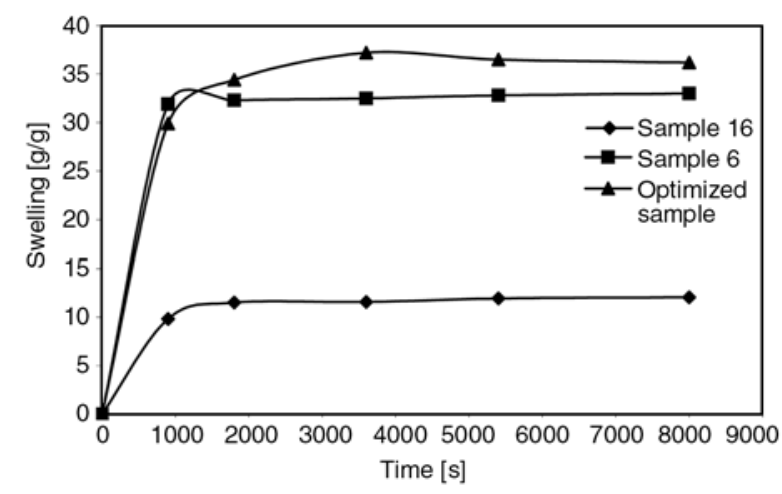

Figure 3. Time dependence of the AUL values for the different water absorbance. capacities (highest absorbency (optimized final product), medium absorbency (sample 6), and the lowest absorbency (sample16) swollen in saline solution (Under pressure $6205 \mathrm{~Pa}$ )

Table 4. Experimental results for equilibrium swelling (ES) capacity

\begin{tabular}{|l|c|c|c|c|c|c|c|c|c|c|c|c|c|c|c|c|}
\hline Trial number & $\mathbf{1}$ & $\mathbf{2}$ & $\mathbf{3}$ & $\mathbf{4}$ & $\mathbf{5}$ & $\mathbf{6}$ & $\mathbf{7}$ & $\mathbf{8}$ & $\mathbf{9}$ & $\mathbf{1 0}$ & $\mathbf{1 1}$ & $\mathbf{1 2}$ & $\mathbf{1 3}$ & $\mathbf{1 4}$ & $\mathbf{1 5}$ & $\mathbf{1 6}$ \\
\hline ES $[\mathrm{g} / \mathrm{g}]$ & 96 & 171 & 205 & 289 & 95 & 94 & 239 & 101 & 115 & 43 & 65 & 85 & 39 & 59 & 31 & 30 \\
\hline
\end{tabular}


of charges in the SAP structure causes an osmotic pressure difference between the gel and the solvent phase. This pressure difference produces a strong driving force to diffuse solvent to the gel phase. The diffusion process continues until the osmotic pressure difference becomes zero. The osmotic pressure difference is reduced in salt solutions, which leads to less swelling in comparison with distilled water. Solvent acts like a plasticizer for the polymer. It is well known that the higher the plasticizer content, the lower the elastic modulus will be.

\subsection{Scanning electron microscopy}

Hydrogels water absorbency and its retention rate depend on hydrogel porosity and mean pore size. Hence, one of the most important properties, which should be considered, is hydrogel microstructure morphologies. This porous micro structure brings about an increased surface area and capillary effect [26]. Figure 4 shows the SEM photographs of CMC and optimized final product. These pictures verify

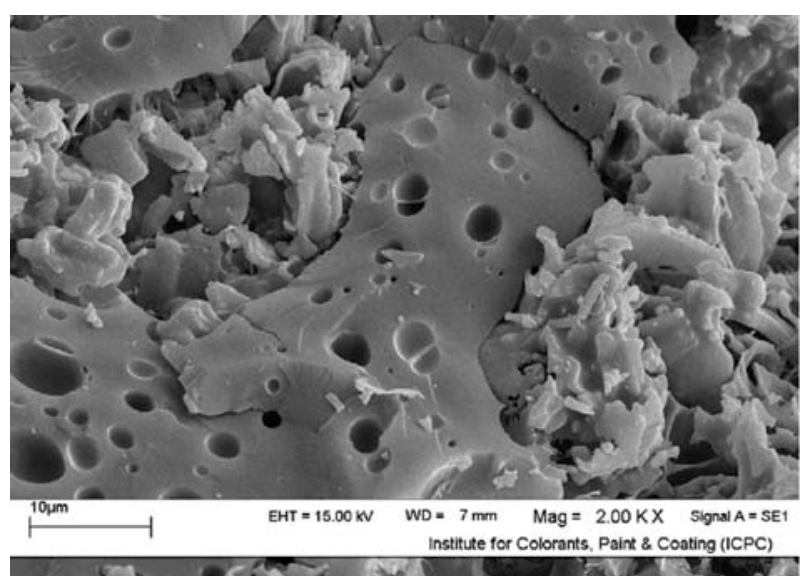

a)

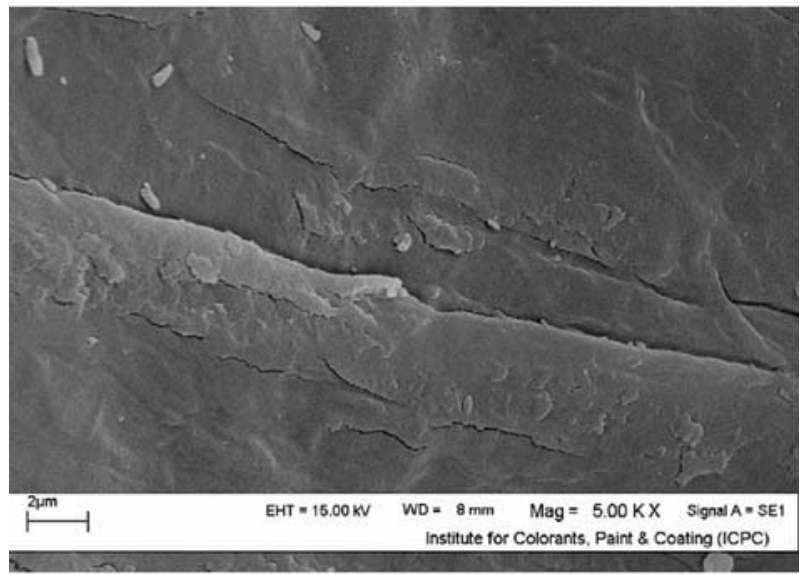

b)

Figure 4. SEM photographs of optimized final product (a) and CMC (b) that graft copolymers have a porous structure. It is supposed that these pores are the regions of water permeation and interaction sites of external stimuli with the hydrophilic groups of the graft copolymers.

\section{Conclusions}

In this work, a novel composite hydrogel, CMC-gpoly (acrylic acid)/Celite composite was prepared by graft copolymerization of AA onto CMC backbones in the presence of a crosslinking agent and powdery Celite. The synthesis conditions of superabsorbent hydrogel were optimized by Taguchi method. The maximum water absorbency $(310 \mathrm{~g} / \mathrm{g})$ was achieved among the different variables; the MBA concentration had the greatest effect on the results. The Taguchi method was found to be promising tool to obtain the optimum conditions for such studies. The absorbency under load (AUL) data is usually given in the patent literature and technical data sheets offered by industrial SAP manufacturers. Swelling capacity of composite SAPs (absorbed saline solution under pressure of $6205 \mathrm{~Pa}$ ) was measured at $25^{\circ} \mathrm{C}$.

\section{References}

[1] Buchholz F., Graham T.: Modern superabsorbent polymer technology. Wiley, New York (1998).

[2] Kudel V.: Encyclopedia of polymer science and engineering. Wiley, NewYork (1985).

[3] Pó R.: Water-absorbent polymers: A patent survey. Journal of Macromolecular Science-Polymer Review, 34, 607-662 (1994).

[4] Lee W-F., Yang L-G.: Superabsorbent polymeric materials. XII. Effect of montmorillonite on water absorbency for poly (sodium acrylate) and montmorillonite nanocomposite superabsorbents. Journal of Applied Polymer Science, 92, 3422-3429 (2004).

[5] Kabiri K., Zohuriaan-Mehr M.: Porous superabsorbent hydrogel composites: synthesis, morphology and swelling rate. Macromolecular Materials and Engineering, 289, 653-661 (2004).

[6] Li A., Wang A.: Synthesis and properties of claybased superabsorbent composite. European Polymer Journal, 41, 1630-1637 (2005).

[7] Zhang J., Chen H., Wang A.: Study on superabsorbent composite. III. Swelling behaviors of polyacrylamide/attapulgite composite based on acidified attapulgite and organo-attapulgite. European Polymer Journal, 41, 2434-2435 (2005). 
[8] Pourjavadi A., Amini-Fazl M. S., Ayyari M.: Taguchi optimized synthesis of collagen-g-poly (acrylic acid)/ kaolin composite superabsobent hydrogel. European Polymer Journal, submitted (2007).

[9] Wu J., Wei Y., Lin J., Lin S. B.: Study on starch-graftacrylamide/mineral powder superabsorbent composite. Polymer, 44, 6513-6520 (2003).

[10] Lin J., Wu J., Yang Z., Pu M.: Synthesis and properties of poly (acrylic acid)/mica superabsorbent nanocomposite. Macromolecular Rapid Communications, 22, 422-424 (2001).

[11] Lee W-F., Chen Y-C.: Effect of intercalated reactive mica on water absorbency for poly (sodium acrylate) composite superabsorbents. European Polymer Journal, 41, 1605-1612 (2005).

[12] Santiago F., Mucientes A., Osorio M., Rivera C.: Preparation of composites and nanocomposites based on bentonite and poly(sodium acrylate). Effect of amount of bentonite on the swelling behaviour. European Polymer Journal, 43, 1-9 (2007).

[13] Wu J., Lin J., Zhou M., Wei C.: Synthesis and properties of starch-graft-polyacrylamide/clay superabsorbent composite. Macromolecular Rapid Communications, 21, 1032-1034 (2000).

[14] Roy R. K.: Design of experiments using the Taguchi approach. Wiley, New York (2001).

[15] Garcia-Diaz A., Philips D.: Principles of experimental design and analysis. Chapman and Hall, London (1995).

[16] Montgomery D. C.: Design and analysis of experiments. Wiley, New York (2001).
[17] Johnson R.: Miller and Freund's probability and statistics for engineers. Prentice-Hall of India, New Delhi (2001).

[18] Ross P.: Taguchi techniques for quality engineering. McGraw-Hill, New York (1989).

[19] Qin J.: Process for the preparation of modified polysaccharides having improved absorbent properties. US Patent 5470964, USA (1995).

[20] Ramazani-Harandi M., Zohuriaan-Mehr M., Yousefi A., Ershad-Langroudi A., Kabiri K.: Rheological determination of the swollen gel strength of superabsorbent polymer hydrogels. Polymer Testing, 25, 470 474 (2006).

[21] Kabiri K., Zohuriaan-Mehr M.: Superabsorbent hydrogel composites. Polymers for Advanced Technologies, 14, 438-444 (2003).

[22] Ma S., Liu M., Chen Z.: Preparation and properties of a salt-resistant superabsorbent polymer. Journal of Applied Polymer Science, 93, 2532-2541 (2004).

[23] Qi X., Liu M., Chen Z., Liang R.: Preparation and properties of diatomite composite superabsorbent. Polymers for Advanced Technologies, 18, 184-193 (2007).

[24] Khraisheh M. A. M., Allenb S. J., Al-Ghouti M. A., Ahmadb M. N.: Effect of $\mathrm{OH}$ and silanol groups in the removal of dyes from aqueous solution using diatomite. Water Research, 39, 922-932 (2005).

[25] Flory P. J.: Principles of polymer chemistry. Cornell University Press, New York (1953).

[26] Lim D-W., Yoon K-J, Ko S-W.: Synthesis of AAbased superabsorbent interpenetrated with sodium PVA sulfate. Journal of Applied Polymer Science, 78, 2525-2532 (2000). 